\title{
Dendritic cell control of immune responses
}

\author{
Penelope A. Morel * and Lisa H. Butterfield \\ University of Pittsburgh, Pittsburgh, PA, USA \\ ${ }^{*}$ Correspondence: morel@pitt.edu \\ Edited by: \\ Claudia Kemper, King's College London, UK \\ Reviewed by: \\ Sandra Stephanie Diebold, King's College London, UK
}

Keywords: dendritic cells, immuno response, autoimmune diseases, inflammation, cancer vaccines

Dendritic cells (DC) play a critical role in the initiation of the immune response, acting as sentinels in the tissues, reacting to invading pathogens and then inducing the activation and differentiation of naïve T cells. Since their discovery by Ralph Steinman in the 1970s, we have learned a great deal about DC biology and function. Distinct DC subsets exist in specific tissue niches and within the secondary lymph nodes, and the study of the phenotype and function of DC subsets in mice and humans has been an area of great interest. DC influence the immune response by directing the differentiation of $\mathrm{T}$ cells into functional subtypes important for elimination of the relevant pathogen. DC also contribute to disease pathogenesis, such as autoimmunity and cancer, through failures in self-tolerance and promotion of an immunosuppressive environment, respectively. In addition, because of their role in molding $\mathrm{T}$ cell responses, $\mathrm{DC}$ have been tested in therapeutic settings in both autoimmunity and cancer. In this research topic, 11 articles cover many aspects of current DC biology, ranging from the classification and function of DC subsets, roles of DC in disease pathogenesis to current use of DC in the therapeutic setting.

The topic begins with two reviews of normal DC function; one is focused on the important topic of cross-presentation (1) and the other on human plasmacytoid (p)DC (2). Cross-presentation is the means by which DC are uniquely able to take up, process, and present exogenous antigens in MHC class I molecules and this review (1) describes novel findings on the role of intracellular vesicular traffic in this process and how it is influenced by inflammatory signals. pDCs were first identified as type 1 interferon-producing cells following acute viral infection and have also been shown to have tolerogenic properties. The review by Mathan et al. (2) focuses on interactions between human pDCs and other cells of the immune system with a particular emphasis on cell surface proteins that facilitate these interactions.

The next four articles in this research topic are original research focusing on various aspects of DC function in vivo and in vitro (3-6). DC maturation is induced by interaction with pathogenderived molecules such as LPS, but infections also induce production of a large number of cytokines. Work by Hartmann et al. (3) examined the effects of virally induced cytokines on the maturation and function of human DC. These studies identified a set of five cytokines that are critical for the induction DC maturation. In addition, this study emphasizes a systems approach to studying the complex effects of cytokine-induced DC maturation (3). DC play an important role in maintaining tolerance to self-antigens and the second article in this section describes the role of early IL-10 production in induction of tolerance to a self-antigen (6). This article builds on the intriguing observation by the same group that immunization with foreign antigen induces a rapid upregulation of pancreatic enzymes in splenic DC that is correlated with the induction of immunity. The present article shows that immunization with a self-antigen fails to induce pancreatic enzymes and demonstrates a role for IL-10 in this phenomenon (6). Work by Kokulus et al. (5) demonstrates the influence of mild chronic cold stress on the phenotype and function of DC in normal and tumorbearing mice. These studies highlight the importance of regulating ambient temperature when conducting experiments with experimental animals and the impact of non-physiologic temperature. Chronic human inflammatory diseases are often characterized by changes in circulating monocyte and DC populations. The final article in this section describes a novel flow cytometry panel, using $\mathrm{CD} 4$ as a lineage marker that allows the enumeration of monocyte, DC, and lymphocyte populations in a single panel (4). This panel was validated in patients with immunodeficiency, cancer, and inflammatory conditions.

The final group of five reviews highlights the role of DC in either causing or ameliorating disease (7-11). Autoimmune diseases are characterized by a breakdown of self-tolerance followed by an immune response that causes damage to normal tissues. DC play roles at all stages of the autoimmune response and these are outlined in a review that focuses specifically on type 1 diabetes (T1D) (11). This review also highlights the potential for using DC to prevent or treat T1D and discusses the latest clinical trials using or targeting DC in this disease (11). The second review focuses on the many types of immunoregulatory DC and their role in preventing inflammatory conditions such as autoimmunity, transplant rejection, and atopic diseases (9). The last three reviews in this research topic focus on the therapeutic use of DC as cancer vaccines. In the first of these reviews, the importance of tertiary lymphoid structures in the development of effective anti-tumor immunity is discussed (8). The authors show that injection of DC, expressing the transcription factor Tbet, into the tumor stimulates the generation of tertiary lymphoid structures and contributes to tumor eradication. The second provides an overview of the preparation, functional characteristics, and use of human DC in cancer vaccines, with particular emphasis on the culture methods, maturation cocktails, antigen formulation, and routes of delivery that are currently in use (7). The final review in this research topic 
focuses on the barriers to the generation of effective DC vaccines in cancer patients (10). In particular, this review outlines the challenges posed by immunosuppressive monocyte populations, prevalent in cancer patients, which impede the generation of immunostimulatory DC vaccines.

Thus, this research topic provides a timely overview of some of the recent advances in DC biology and we look forward to many new developments in this exciting field.

\section{ACKNOWLEDGMENTS}

We would like to thank all authors for their contributions to this research topic.

\section{REFERENCES}

1. Nair-Gupta P, Blander JM. An updated view of the intracellular mechanisms regulating cross-presentation. Front Immunol (2013) 4:401. doi:10.3389/fimmu. 2013.00401

2. Mathan TS, Figdor CG, Buschow SI. Human plasmacytoid dendritic cells: from molecules to intercellular communication network. Front Immunol (2013) 4:372. doi:10.3389/fimmu.2013.00372

3. Hartmann BM, Marjanovic N, Nudelman G, Moran TM, Sealfon SC. Combinatorial cytokine code generates anti-viral state in dendritic cells. Front Immunol (2014) 5:73. doi:10.3389/fimmu.2014.00073

4. Jardine L, Barge D, Ames-Draycott A, Pagan S, Cookson S, Spickett G, et al. Rapid detection of dendritic cell and monocyte disorders using CD4 as a lineage marker of the human peripheral blood antigen-presenting cell compartment. Front Immunol (2013) 4:495. doi:10.3389/fimmu.2013.00495

5. Kokolus KM, Spangler HM, Povinelli BJ, Farren MR, Lee KP, Repasky EA. Stressful presentations: mild cold stress in laboratory mice influences phenotype of dendritic cells in naive and tumor-bearing mice. Front Immunol (2014) 5:23. doi:10.3389/fimmu.2014.00023
6. Marvel DM, Finn OJ. Global inhibition of DC priming capacity in the spleen of self-antigen vaccinated mice requires IL-10. Front Immunol (2014) 5:59. doi:10.3389/fimmu.2014.00059

7. Butterfield LH. Dendritic cells in cancer immunotherapy clinical trials: are we making progress? Front Immunol (2013) 4:454. doi:10.3389/fimmu.2013.00454

8. Chen L, Fabian KL, Taylor JL, Storkus WJ. Therapeutic use of dendritic cells to promote the extranodal priming of anti-tumor immunity. Front Immunol (2013) 4:388. doi:10.3389/fimmu.2013.00388

9. Gordon JR, Ma Y, Churchman L, Gordon SA, Dawicki W. Regulatory dendritic cells for immunotherapy in immunologic diseases. Front Immunol (2014) 5:7. doi:10.3389/fimmu.2014.00007

10. Laborde RR, Lin Y, Gustafson MP, Bulur PA, Dietz AB. Cancer vaccines in the world of immune suppressive monocytes (CD14(+)HLA-DR(lo/neg) cells): the gateway to improved responses. Front Immunol (2014) 5:147. doi:10.3389/ fimmu.2014.00147

11. Morel PA. Dendritic cell subsets in type 1 diabetes: friend or foe? Front Immunol (2013) 4:415. doi:10.3389/fimmu.2013.00415

Conflict of Interest Statement: The authors declare that the research was conducted in the absence of any commercial or financial relationships that could be construed as a potential conflict of interest.

Received: 24 December 2014; accepted: 22 January 2015; published online: 05 February 2015.

Citation: Morel PA and Butterfield LH (2015) Dendritic cell control of immune responses. Front. Immunol. 6:42. doi: 10.3389/fimmu.2015.00042

This article was submitted to Molecular Innate Immunity, a section of the journal Frontiers in Immunology.

Copyright (C) 2015 Morel and Butterfield. This is an open-access article distributed under the terms of the Creative Commons Attribution License (CC BY). The use, distribution or reproduction in other forums is permitted, provided the original author(s) or licensor are credited and that the original publication in this journal is cited, in accordance with accepted academic practice. No use, distribution or reproduction is permitted which does not comply with these terms. 